89

\title{
A Concept Model for Maintaining Awareness of Document Configuration
}

\author{
Shi Tao \\ UNSECO Supported International Centre for Engineering Education (USICEE) \\ Monash University, VIC 3168, Australia \\ tao@eng.monash.edu.au
}

KEY WORDS: CSCW

$\mathrm{CSCW}$ is essentially a cooperative process and involves bringing expertise together in order to develop a document. The document components are worked on by collaborators independently and are assembled and reassembled based on the requirements of the working system. In order to ensure consistency within the document in a changing environment it is necessary to maintain awareness of document configuration among individual collaborators. In this poster, a multi-dimensional model is presented as a first step to explicitly represent the complex relationships among the components in the collaborative document preparation, with the objective of maintaining awareness of document configuration in asynchronous distributed cooperation. This proposal is primarily based on Prof. Igor Hawryszkiewycz' paper(Hawryszkiewycz, 1996). The prototype will be developed with Java.

The first dimension of the model shown below is project workflow. In general, the task sequence is neither linear nor parallel, but exhibits network behaviour with change being dynamic. Each task in the process is linked by a set of vectors which can be seen as the function of time. This means that the same task can be repeated at different times. This dimension is project oriented with the aim of producing the relevant document.

The second dimension consists of three layers. The first layer is the collaborative document preparation. It is a set of document preparation activities which are affiliated to each project task. The activities consist of task-cycles, and each cycle has several steps. It is document management oriented. The second layer represents the document's various states distinguished by different colours. The document evolves through the task-cycle as it progresses from one state to the next. The task-cycle steps can be mapped into document management at each state transition. The document states can, therefore, be links between tasks and document components, which can be used to model dynamics. The third layer is about versioning policies. The versioning policy can be seen as an integrative mechanism at conceptual level to combine activities with artefacts. Each of the version numbers correspond to certain activities during document preparation. The number changes should automatically be displayed to individuals. The versioning policy consists of two parts - coordination and private. The coordination policy is defined in terms of configuration, task-cycle and cycle-step. Each time

Human-Computer Interaction: INTERACT'97 S. Howard. J. Hammond \& G. Lindgaard (editors)

Published by Chapman \& Hall CIFIP 1997 


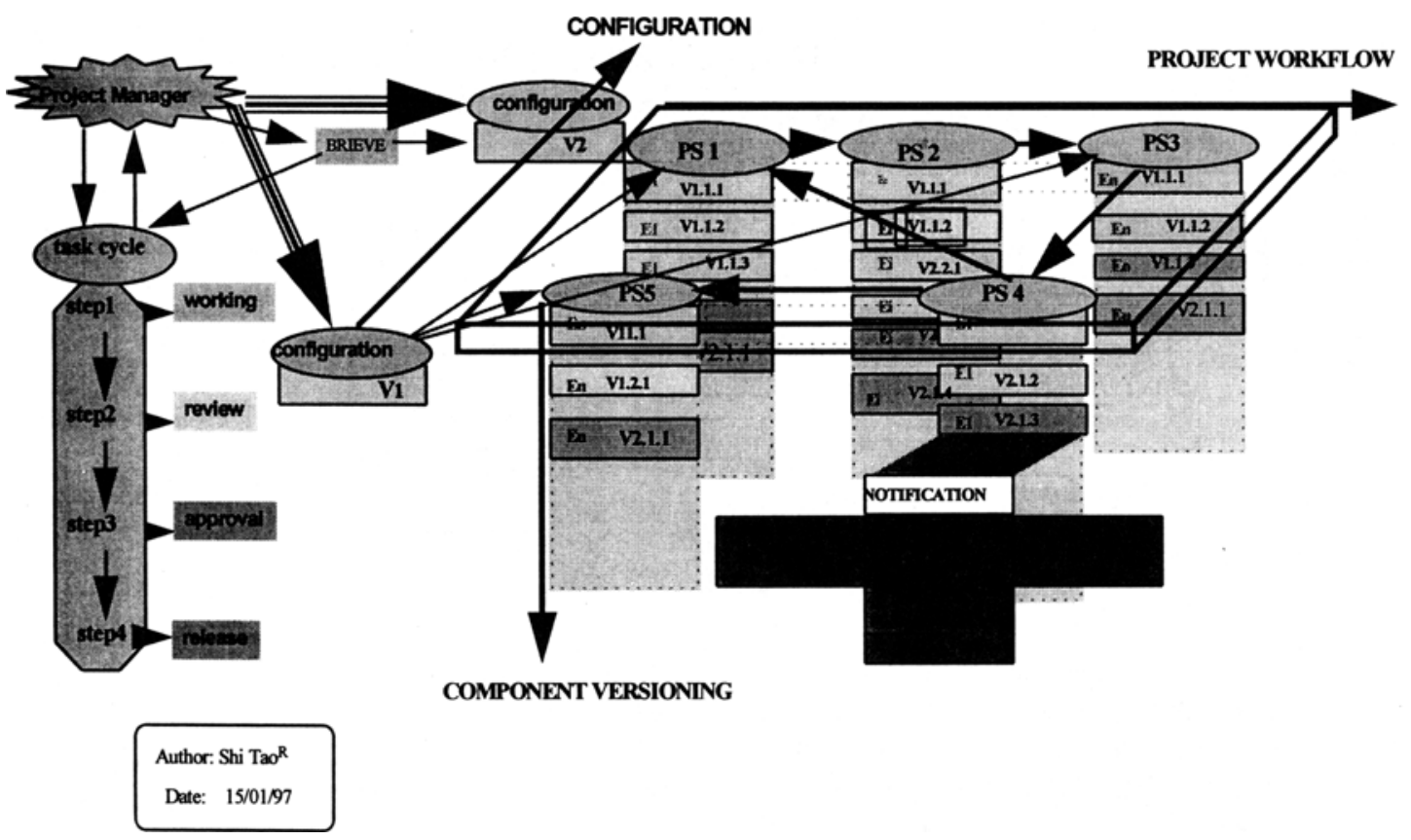

Figure 1: A multi-dimensional concept model.

a new configuration is constituted, the first number of the version in this part is changed and a new brief may be issued. Once the document goes through a task-cycle, the second number of the version is incremented. Except for step one, any task-cycle step can change the third number of the version. The private policy is a set of individual versioning conventions. The changes made by individual writers are hidden from all other collaborators.

The third dimension of the model is configuration. The configuration is meaningful compositions of document versions in a given state consistent with its ontology. The ontology is a set of specifications of physical elements at the semantic level. It is like a set of IP addresses acting as an invisible assembly line embedded in the document elements. For each configuration, the relevant components are combined and their first version numbers are changed. As the components are grouped and invoked, individuals are notified. This can be done by blinking the component in the model and informing the individual collaborator of the occurrence. Notification with a detailed description of the changes can be accessed by clicking the component nodes.

\section{References}

1. Hawryszkiewycz, I.T., An architecture for supporting asynchronous processes. (Unpublished) (1996). 